\title{
Bidirectional Symmetric 25G Coherent ONU Using a Single Laser, Single-ended PIN and a 2-bit ADC
}

\author{
M. Sezer Erkılınç, Zhixin Liu, Thomas Gerard, Robert I. Killey, Polina Bayvel, and Domaniç Lavery
}

Optical Networks Group, Dept. of Electronic \& Electrical Engineering, University College London, UK m.erkilinc@ee.ucl.ac.uk (Email of corresponding author mandatory)

Abstract We realize a coherent ONU using only a single PIN phododiode, a 2-bit ADC, and an EML shared signal/LO-laser. The receiver sensitivity after $20 \mathrm{~km}$ transmission is enhanced by $18.6 \mathrm{~dB}$ over direct detection PAM-4, but requires no additional optoelectronic hardware.

\section{Introduction}

The conventional approach to signal transmission in optical access networks is reaching a saturation point. As seen by the recent developments in the 100G-EPON standardization process, the line rates, at least in the short term, will be set at $25 \mathrm{Gbit} / \mathrm{s}$, with wavelength multiplexing used to increase the rate per passive optical network (PON) to $100 \mathrm{Gbit} / \mathrm{s}$. This constraint is, in part, due to both the increased electronic bandwidth requirements for systems operating beyond $25 \mathrm{Gbit} / \mathrm{s}$, and the reduced chromatic dispersion tolerance when operating at high symbol rates. To overcome this, multi-level modulation formats, such as duobinary and 4-ary pulse amplitude modulation (PAM-4) are now being considered. However, due to the increased power loss from directly modulated PAM- 4 versus NRZ, the use of externally modulated lasers (EML) has been proposed. A prototypical PAM-4 optical network unit (ONU) thus comprises an EML, a single PIN photodiode, and 4-level logic for demodulation.

It was recently shown that a coherent receiver using three PIN photodiodes offers coherent gain of almost $20 \mathrm{~dB}$ over NRZ at $25 \mathrm{Gbit} / \mathrm{s}$, and may be suitable for deployment in an optical line terminal $(\mathrm{OLT})^{1}$. However, the requirement for a separate local oscillator (LO) laser and three photodiodes means such a technique remains unattractive for use in an ONU. This raises the question: Is it possible to realise coherent detection for a $25 \mathrm{G}$ PON using the components from a PAM-4 ONU?

In this paper, we definitively answer this question by using simple digital signal processing (DSP) algorithms to enable a polarizationindependent coherent ONU based on hardware found in a typical PAM-4 ONU. Moreover, we demonstrate the feasibility of using a shared ONU laser for burst mode upstream/LO downstream, and demonstrate that linear detection is preserved.

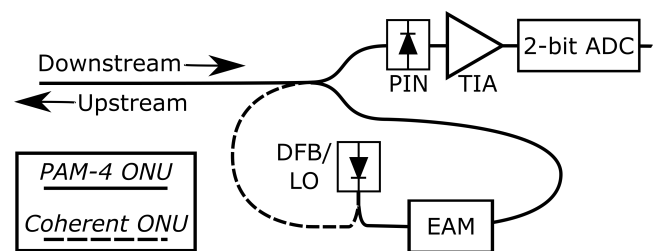

Fig. 1: An ONU for generation, and direct detection-based reception, of optical PAM-4 signals. The dashed path converts the receiver from direct detection to coherent ${ }^{2}$.

Redesign of a PAM-4 ONU as a Coherent ONU A conventional ONU uses a single PIN photodiode with time-division multiplexed on-off keying (TDM-OOK) for upstream transmission. For bandwidth-efficiency and dispersion tolerance at higher data rates, PAM- 4 for example, could be transmitted using an electro-absorption modulator (EAM), and received using a single PIN photodiode with 4-level logic; i.e., a 2-bit electronic demodulator, or analogue-to-digital converter (ADC). An ONU with the capability to transmit and receive PAM- 4 signals is shown, schematically, in Fig. 1 (solid paths).

Coherent receivers require a phase reference signal, provided by a LO laser. Unlike the directly modulated laser used for TDM-OOK, the signal from an EML can be divided, either using a splitter between the laser diode and the EAM, or by engineering the laser to emit a small amount of signal from the rear facet. Fig. 1 (dashed paths) shows a method for dividing the power between the upstream and downstream, in order to provide both a TDM amplitude modulated signal for the upstream, and a continuous wave signal for the LO. If the same 4-level logic is used in the receiver, then there is no additional complexity in the ONU for this receiver versus the direct detection PAM-4 ONU, other than for the inclusion of DSP. There are several low complexity methods, such as polarization-switching or Alamouti coding, which can be applied to the downstream signal at the optical line terminal (OLT) to provide polarization independence at the $\mathrm{ONU}^{3,4}$. 


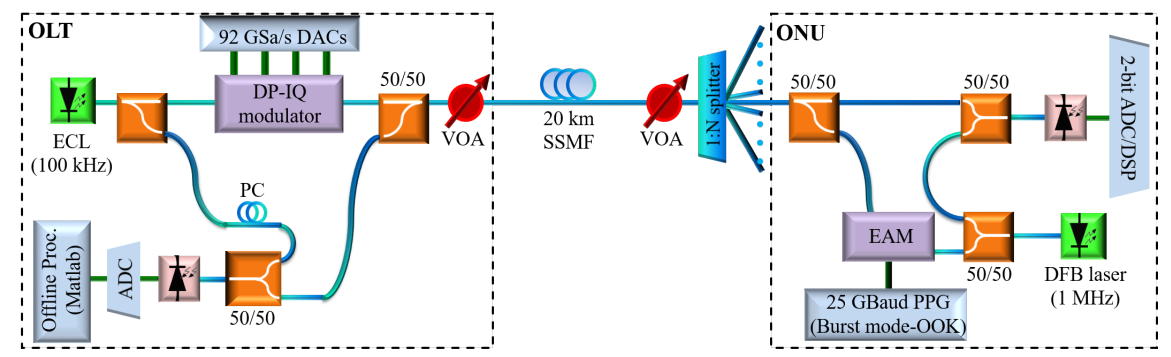

Fig. 2: Experimental configuration. The ONU (right) shares its EML between upstream/downstream. For experimental convenience, an additional $2 \times 1$ power divider was included at the input of the ONU; this has no impact on the receiver sensitivity measurement. Key: PC: Polarization controller, VOA: Variable optical attenuator.

\section{Experimental Configuration}

The experimental configuration is shown in Fig. 2. For this work, we chose to demonstrate the operation of the simplified ONU shown in Fig. 1 by modulating an Alamouti-coded downstream signal at the OLT. To generate this signal, the $1550 \mathrm{~nm}$ wavelength output from an external cavity laser (ECL) was passed to a dual polarization (DP) IQ modulator. The modulator was driven using four electrical signals constructed to generate a root raised cosine (RRC, 0.1 roll-off) shaped quadrature phase shift keying (QPSK) signal at $12.5 \mathrm{GBd}$. The two polarization arms were precoded to generate an Alamouti code (to enable signal recovery without a polarization diverse receiver ${ }^{4}$ ), and orthogonally polarized pilot tones were inserted with a $500 \mathrm{MHz}$ frequency separation from the stop band of the signal to simplify equalization and phase recovery at the receiver.

This signal was transmitted downstream over $20 \mathrm{~km}$ standard single mode fiber (SSMF), and was coherently received using a single-ended PIN photodiode. This required a LO; here provided by a distributed feedback laser (DFB), linewidth $1 \mathrm{MHz}$, and output power $+13 \mathrm{dBm}$. The DFB output was divided between upstream and downstream, leaving a power of $+6 \mathrm{dBm}$ to be used for the LO. Heterodyne detection requires the LO to be frequency offset from the signal by at least half the symbol rate $(6.25 \mathrm{GHz})$, and in practice we used a frequency offset of approximately $10 \mathrm{GHz}$ to avoid the low frequency noise terms introduced due to direct detection of signalsignal beating. The electrical signal from the photodiode was amplified, and subsequently digitized using a sampling oscilloscope, and resampled to $4 \mathrm{Sa} / \mathrm{symbol}$. An additional quantization stage was then applied to the signal, emulating an ADC. The pilot tones were used to digitally downconvert the signal to baseband and recover the carrier phase, and signal equalization was applied using a combination of chromatic dispersion compensation and blind adaptive filtering based on the constant modulus algorithm.

For upstream transmission, the second half of the DFB laser output modulated with $25 \mathrm{Gbit} / \mathrm{s}$ TDM-OOK (40 $\mu$ s on-off burst) using an EAM. The upstream signal was then coupled into the fiber path using the second arm of the $2 \times 2$ coupler (which was originally used to combine the downstream signal and the LO). The signal was, again, received using a single PIN photodiode, although, as in ${ }^{1}$, a manual polarization controller was used to measure the upstream signal. In practice, either adaptive polarization control would be required, or a polarization independent receiver could be used for a $3 \mathrm{~dB}$ sensitivity penalty ${ }^{5}$. For this receiver, the $L O$ power was $+9 \mathrm{dBm}$; which, notably, is lower than the $+12 \mathrm{dBm}$ used in the demonstration in ${ }^{1}$, and required no additional laser source at either the ONU or the OLT.

\section{Results and Discussion}

The back-to-back characterisation of the downstream signal is shown in Fig. 3(a). When the 6bit $A D C$ resolution is used, the receiver sensitivity at a bit error ratio (BER) of $10^{-3}$ is $-31.6 \mathrm{dBm}$. This represents an excellent receiver sensitivity versus many direct detection systems, despite including a $3 \mathrm{~dB}$ penalty due to heterodyne detection, and an additional $3 \mathrm{~dB}$ penalty due to the rate- $\frac{1}{2}$ code used to achieve polarization independence. No penalty was observed when restricting the ADC resolution to 4-bits. For the 3-bit and 2-bit ADCs, we introduced a clipping ratio of 0.45 in order to make best use of the 8- and 4levels available, respectively. For this configuration, the receiver sensitivity was measured to be $-30.9 \mathrm{dBm}$ and $-27.0 \mathrm{dBm}$, respectively.

After transmission over $20 \mathrm{~km}$ SSMF, no penalty was observed when using $\geq 3$-bits for the ADC, despite operating in the C-band. The 2-bit quantized signal suffered a $0.4 \mathrm{~dB}$ penalty after transmission (as shown in Fig. 3(b)), which can be attributed to the additional quantization levels required to accurately represent a dispersed signal. 

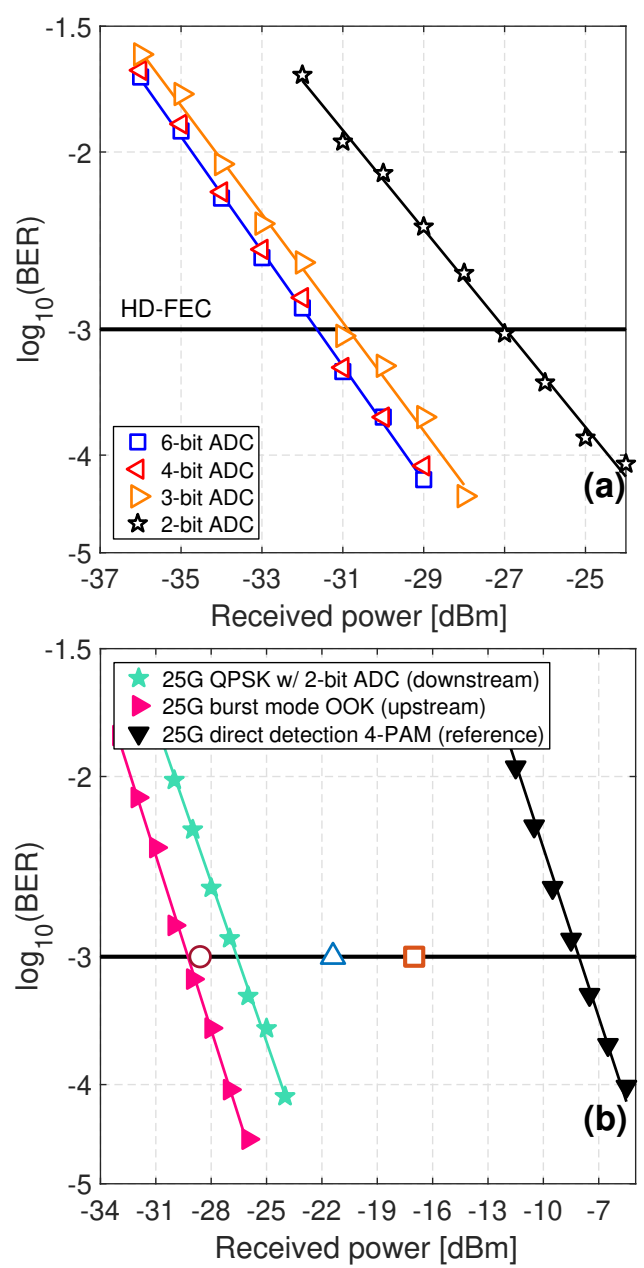

Fig. 3: Receiver sensitivity for (a) back-to-back Alamouti coded QPSK signal, and (b) after transmission over $20 \mathrm{~km}$ upstream/downstream. The open markers shown in (b) represent state-of-the-art reference points for a $25 \mathrm{Gbit} / \mathrm{s}$ APD receiver with OOK (circle) and duobinary (triangle), and PIN-detection OOK (square) ${ }^{\dagger}$. A reference direct detection PAM-4 curve is also shown, taken using the same PIN as used for the coherent system.

The 2-bit result is comparable with the sensitivity achieved for back-to-back 25 Gbit/s TDM-OOK for an avalanche photodiode (APD) ${ }^{1}$, and is $10 \mathrm{~dB}$ more sensitive than the PIN photodiode reported in the same work. For completeness, we measured the sensitivity of direct detection $25 \mathrm{Gbit} / \mathrm{s}$ PAM-4 using the same PIN photodiode as in the coherent measurements. The coherent QPSK system outperforms PAM-4 by $18.6 \mathrm{~dB}$. It should be noted that, for the case of coherent detection, chromatic dispersion can be compensated digitally in the ONU, meaning that transmission beyond $20 \mathrm{~km}$ is feasible for this configuration. Despite the upstream signal and the LO sharing a $2 \times 2$ coupler for fibre ingress/egress, the reflections from the upstream signal caused no detrimental effect on the measured downstream signal (see Fig. 2); in part due to the frequency selectivity of the coherent receiver, and the inherent frequency offset between upstream and downstream signals when using this configuration. For this reason, the ONU configuration described is inherently suitable for wavelength division multiplexed operation.

To complete the proof-of-principle demonstration, the upstream signal (25 Gbit/s TDM-OOK) was measured using a single PIN receiver, and the results are shown in Fig. 3(b). Due to the coherent gain offered by the LO laser, the receiver sensitivity was measured to be $-29.3 \mathrm{dBm}$. The use of an adaptive equaliser at the receiver meant that there was no penalty observed due to EAM chirp from modulation or burst mode operation.

\section{Conclusions}

A $25 \mathrm{Gbit} / \mathrm{s}$ coherent ONU was demonstrated using only equipment required by a direct detection PAM-4 ONU. This proof-of-principle experiment showed that, through the use of low complexity receiver-side DSP, the limitations imposed by a single PIN receiver and a 2-bit ADC could be overcome. This demonstration showed that an EML could, practically, be modified to be used for simultaneous upstream/downstream operation, and that there is sufficient power available from a conventional DFB to achieve this. Reflections from bidirectional operation were not found to impact the transmission performance, and the linearity of the receiver was preserved; with $20 \mathrm{~km}$ chromatic dispersion compensated digitally.

\section{Acknowledgements}

This work was supported by a Royal Academy of Engineering Research Fellowship. Support from Huawei Technologies is gratefully acknowledged.

\section{References}

[1] D. van Veen and V. Houtsma, "Bi-directional 25G/50G TDM-PON with Extended Power Budget using 25G APD and Coherent Amplification," paper Th5A.4 in Proc. Optical Fiber Communication Conference 2017.

[2] D. Lavery et al. "Recent progress and outlook for coherent PON," paper M3B.1 in Proc. Optical Fiber Communication Conference 2018.

[3] I. N. Cano et al., "Polarization independent single-PD coherent ONU receiver with centralized scrambling in udWDM-PONs," paper P.7.12 in Proc. European Conference on Optical Communications 2014.

[4] M. S. Erkılınç et al., "Polarization-insensitive single balanced photodiode coherent receiver for passive optical networks," paper Th.1.3.3 in Proc. European Conference on Optical Communication 2015.

[5] B. Glance, "Polarization independent coherent optical receiver," J. Lightw. Technol. 5 (2) pp. 274-276 (1987). 